\title{
Atypische Beschäftigungsverhältnisse. Stand und Lücken der aktuellen Diskussion
}

Atypische Beschäftigungsverhältnisse nehmen zu. Diese Entwicklung wäre weniger besorgniserregend, wenn sie nicht mit hohen Prekaritätsrisiken verbunden wäre. Geringere Löhne, schlechter Zugang zu betrieblicher Weiterbildung und höhere Beschäftigungsinstabilität wiegen umso schwerer, als der Aufstieg aus atypischen in Normalarbeitsverhältnisse erheblich eingeschränkt ist. Die sozialen Risiken verlängern sich in die Nacherwerbsphase. Einem wachsenden Personenkreis droht Armut im Alter. Der Beitrag liefert einen Überblick über Ausmaß und Entwicklung atypischer Beschäftigung und zeigt den Forschungsstand zu den hiermit verbundenen Prekaritätsrisiken auf.

\section{Problemstellung}

Atypische Beschäftigungsverhältnisse sind kein neues Phänomen. Vermehrte Beachtung in der wissenschaftlichen und politischen Debatte finden sie seit den „Gesetzen für moderne Dienstleistungen am Arbeitsmarkt" (im Folgenden: Hartz-Gesetze), die 2003/04 den rechtlichen Rahmen für Leiharbeit sowie für befristete und geringfügige Beschäftigung lockerten, um deren Einsatz und damit das Beschäftigungsniveau insgesamt zu fördern. Schon 2001 hatte das Teilzeit- und Befristungsgesetz die Weichen für eine Ausweitung der Teilzeitarbeit gestellt. Angesichts dieser Deregulierungen verwundert es nicht, dass der Anteil atypischer Beschäftigungsformen seitdem deutlich (auf über ein Drittel der Gesamtbeschäftigung) zugenommen hat. Diese Expansion wirft neue Probleme auf, die bei der Deregulierung weitgehend aus dem Blick geraten waren und sich nun in erhöhten Prekaritätsrisiken niederschlagen.

Auch der Informations- und Wissensstand zu verschiedenen Problemen dieser Beschäftigungsformen hat sich in den vergangenen Jahren, u.a. durch die Evaluationsstudien zu den HartzGesetzen, zweifellos deutlich verbessert (BMAS 2006). Allerdings dominieren nach wie vor Studien zu den einzelnen Formen; überblicksartige Darstellungen, welche die Veränderungen des Beschäf- tigungssystems insgesamt und ihre Folgen deutlich machen, sind weiterhin die Ausnahme (Keller/Seifert 1995; 2007). Außerdem stehen zumeist aktuelle Probleme (vor allem Einkommensdifferenzen, Übernahmechancen) im Mittelpunkt; demgegenüber werden die langfristigen sozialpolitischen Konsequenzen, die sowohl auf individueller als auch kollektiver Ebene auftreten, nur selten thematisiert, obwohl sie inzwischen deutlich zutage treten. Damit werden Maßnahmen der (Re-) Regulierung vernachlässigt, ${ }^{1}$ die im $\mathrm{Ge}$ gensatz zur Politik der vergangenen Jahre zum Ziel haben müssten, die Integration in die sozialen Sicherungssysteme und das Niveau der darauf basierenden Leistungen zu verbessern. In diesem Kontext geht es darum, die Grenzen zwischen Arbeits- und Sozialpolitik zu überwinden.

Dieser Beitrag verfolgt drei Ziele. Zum einen liefert er einen knappen Überblick über die Entwicklung der Formen atypischer Beschäftigung, ihren Umfang und ihre sozialstrukturelle Zusammensetzung (Abschnitt 2). Zum anderen geht es darum, den Forschungsstand zu den mit atypischer Beschäftigung verbundenen Prekaritätsrisiken aufzuzeigen (Abschnitt 3) und daraus Erkenntnisse für Re-Regulierungen abzuleiten (Abschnitt 4). Ausgespart bleibt eine Auseinandersetzung mit den wenigen theoretischen Ansätzen (Nienhüser 2007; Sesselmeier 2007; Neubäumer/Tretter 2008), die Erklärungen für die Expansion atypischer Beschäftigung liefern. Ausgeklammert bleibt ferner die Frage nach dem
Zusammenhang von Beschäftigungsniveau und atypischer Beschäftigung.

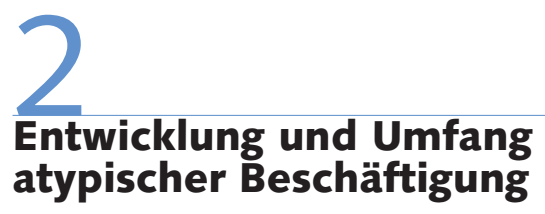

\subsection{EINZELNE FORMEN}

Seit den frühen 1990er Jahren² nehmen sämtliche Formen atypischer Beschäftigung zu, zu denen hier neben der Leiharbeit, den geringfügigen und den befristeten Beschäftigungsverhältnissen auch die sozialversicherungspflichtige Teilzeitarbeit

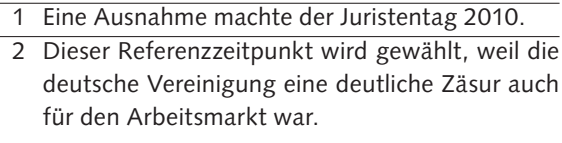
deutsche Vereinigung eine deutliche Zäsur auch für den Arbeitsmarkt war.

Berndt Keller, Prof. em., Dr., bis 2009
Professor für Arbeits- und Sozialpolitik an
der Universität Konstanz. Arbeitsschwer-
punkte: Arbeitspolitik des öffentlichen
Sektors, Folgeprobleme der europäischen
Integration, atypische Beschäftigungsver-
hältnisse, Flexicurity, Zusammenschlüsse
von Gewerkschaften.
e-mail: Berndt.Karl.Keller@uni-konstanz.de
Hartmut Seifert, Dr., ehemaliger Leiter des
Wirtschafts- und Sozialwissenschaftlichen
Instituts (WSI) in der Hans-Böckler-Stiftung.
e-mail: H.G.Seifert@t-online.de


Tabelle 1: Entwicklung der Formen atypischer Beschäftigung

\begin{tabular}{|c|c|c|c|c|c|c|c|c|c|c|c|c|}
\hline \multirow[b]{2}{*}{ Jahr } & \multirow[b]{2}{*}{$\begin{array}{l}\text { Beschäf- } \\
\text { tigte } \\
\text { insgesamt }\end{array}$} & \multicolumn{2}{|c|}{ Teilzeitarbeit $^{11}$} & \multicolumn{4}{|c|}{ Geringfügig Beschäftigte ${ }^{2 / 3)}$} & \multicolumn{2}{|c|}{ Leiharbeit ${ }^{2)}$} & \multirow[b]{2}{*}{$\begin{array}{l}\text { Beschäftigte } \\
\text { (ohne Aus- } \\
\text { zubildende) } \\
\text { insgesamt }\end{array}$} & \multicolumn{2}{|c|}{$\begin{array}{l}\text { Befristet Beschäftigte } \\
\text { (ohne Auszubildende) }\end{array}$} \\
\hline & & in 1.000 & $\begin{array}{l}\text { Anteil an } \\
\text { Beschäf- } \\
\text { tigten } \\
\text { in \% }\end{array}$ & in 1.000 & $\begin{array}{l}\text { Anteil an } \\
\text { Beschäf- } \\
\text { tigten } \\
\text { in \% }\end{array}$ & $\begin{array}{l}\text { Aus- } \\
\text { schließlich } \\
\text { geringfügig } \\
\text { Beschäftige } \\
\text { in } 1.000\end{array}$ & $\begin{array}{l}\text { Anteil an } \\
\text { Beschäf- } \\
\text { tigten } \\
\text { in \% }\end{array}$ & in 1.000 & $\begin{array}{l}\text { Anteil an } \\
\text { Beschäf- } \\
\text { tigten } \\
\text { in \% }\end{array}$ & & in 1.000 & $\begin{array}{c}\text { Anteil an } \\
\text { Beschäftigten } \\
\text { (ohne Auszu- } \\
\text { bildende) } \\
\text { in } \%\end{array}$ \\
\hline 1991 & 33.887 & 4.736 & 14,0 & & & & & 134 & 0,4 & 32.323 & 2.431 & 7,5 \\
\hline 1992 & 33.320 & 4.763 & 14,3 & & & & & 136 & 0,4 & 31.891 & 2.495 & 7,8 \\
\hline 1993 & 32.722 & 4.901 & 15,0 & & & & & 121 & 0,4 & 31.151 & 2.221 & 7,1 \\
\hline 1994 & 32.300 & 5.122 & 15,9 & & & & & 139 & 0,4 & 30.958 & 2.322 & 7,5 \\
\hline 1995 & 32.230 & 5.261 & 16,3 & & & & & 176 & 0,5 & 30.797 & 2.388 & 7,8 \\
\hline 1996 & 32.188 & 5.340 & 16,6 & & & & & 178 & 0,6 & 30.732 & 2.356 & 7,7 \\
\hline 1997 & 31.917 & 5.659 & 17,7 & & & & & 213 & 0,7 & 30.436 & 2.453 & 8,1 \\
\hline 1998 & 31.878 & 5.884 & 18,5 & & & & & 253 & 0,8 & 30.357 & 2.536 & 8,4 \\
\hline 1999 & 32.497 & 6.323 & 19,5 & & & 3.658 & 11,3 & 286 & 0,9 & 30.907 & 2.842 & 9,2 \\
\hline 2000 & 32.638 & 6.478 & 19,8 & & & 4.052 & 12,4 & 339 & 1,0 & 31.014 & 2.744 & 8,8 \\
\hline 2001 & 32.743 & 6.798 & 20,8 & & & 4.132 & 12,6 & 357 & 1,1 & 31.176 & 2.740 & 8,8 \\
\hline 2002 & 32.469 & 6.934 & 21,4 & & & & & 336 & 1,0 & 30.904 & 2.543 & 8,2 \\
\hline 2003 & 32.043 & 7.168 & 22,4 & 5.533 & 17,3 & 4.375 & 13,7 & 327 & 1,0 & 30.513 & 2.603 & 8,5 \\
\hline 2004 & 31.405 & 7.168 & 22,8 & 6.466 & 20,6 & 4.803 & 15,3 & 400 & 1,3 & 29.822 & 2.478 & 8,3 \\
\hline 2005 & 32.066 & 7.851 & 24,5 & 6.492 & 20,2 & 4.747 & 14,8 & 453 & 1,4 & 30.470 & 3.075 & 10,1 \\
\hline 2006 & 32.830 & 8.594 & 26,2 & 6.751 & 20,6 & 4.854 & 14,8 & 598 & 1,8 & 31.371 & 3.389 & 10,8 \\
\hline 2007 & 33.606 & 8.841 & 26,3 & 6.918 & 20,6 & 4.882 & 14,5 & 731 & 2,2 & 31.906 & 3.291 & 10,3 \\
\hline 2008 & 34.241 & 9.008 & 26,3 & 6.792 & 19,8 & 4.882 & 14,3 & 794 & 2,3 & 32.232 & 3.106 & 9,6 \\
\hline 2009 & 34.203 & 9.076 & 26,5 & 6.993 & 20,4 & 4.932 & 14,4 & 610 & 1,8 & 32.558 & 3.026 & 9,3 \\
\hline \multicolumn{13}{|c|}{$\begin{array}{l}\text { 1) jeweils April. } \\
\text { 2) jeweils Ende Juni. } \\
\text { 3) Mini-Jobs auf } 400 € \text { Basis. }\end{array}$} \\
\hline \multicolumn{13}{|c|}{$\begin{array}{l}\text { Quelle: Statistisches Bundesamt, F 1, Reihe 4. 1. 1., verschiedene Jahrgänge und } \\
\text { https://www-ec.destatis.de/csp/shop/sfg/bpm.html.cms.cBroker.cls?cmspath= struktur,sfgsuchergebnis.csp; } \\
\text { Bundesagentur für Arbeit, http://www.pub.arbeitsamt.de/hst/services/statistik/detail/b.html. }\end{array}$} \\
\hline
\end{tabular}

gezählt wird. ${ }^{3}$ Ausgangsniveaus und Dynamik der Entwicklung zeigen deutliche Unterschiede (Tabelle 1). ${ }^{4}$

Ähnlich wie in anderen EU-Mitgliedsländern ist „reguläre“ Teilzeitarbeit (mit mehr als $26 \%$ ) die am weitesten verbreitete Form (Schmid/Protsch 2009). Ihre langfristige, über die Konjunkturzyklen hinweg verlaufende Ausweitung hängt eng mit der zunehmenden Erwerbstätigkeit von Frauen zusammen, die über $80 \%$ der Teilzeitbeschäftigten ausmachen. Überwiegend gehen sie Teilzeitarbeitsverhältnisse „freiwillig" ein, besonders um berufliche und familiäre Aufgaben vereinbaren zu können. Ein knappes Viertel nimmt diese Beschäftigungsform jedoch mangels fehlender Vollzeitarbeit unfreiwillig wahr (destatis 2009).

Ungefähr $20 \%$ arbeiten als geringfügig Beschäftigte. Nach den Änderungen durch die Hartz-Gesetze erfolgte zunächst eine deutliche Zunahme (von weniger als $13 \%$ auf ca. $20 \%$ ); seitdem stagniert die Entwicklung auf hohem Niveau. Wegen der unterschiedlichen Konsequenzen für die soziale Sicherung ist $\mathrm{zu}$ unterscheiden zwischen ausschließlich ausgeübten und Nebenerwerbstätigkeiten, die zusätzlich zu einer sozialversicherungspflichtigen Beschäfti- gung geleistet werden. Die zuerst genannte Variante, die ca. zwei Drittel aller Minijobs umfasst, ist in sozialpolitischer Hinsicht wegen der unzureichenden sozialen Absicherung wesentlich problematischer. ${ }^{5}$

Im Vergleich zu Minijobs ist das Ausmaß (ca. 700.000) der Midijobs deutlich geringer; die Zahl liegt fast in der Größendimension der Leiharbeit.

Befristete Beschäftigung hat trotz verschiedener Deregulierungsschritte auf lediglich ca. $10 \%$ zugenommen. Ursprüngliche Befürchtungen einer massiven Expansion infolge der Deregulierungen sind nicht eingetreten; allerdings sind bestimmte Gruppen (vor allem jüngere Arbeitnehmer beim Eintritt in den Arbeitsmarkt) überproportional häufig betroffen. Von zentraler Bedeutung ist die Frage, ob der Übergang in ein unbefristetes Beschäftigungsverhältnis gelingt.

Leiharbeit $^{6}$ umfasst im Vergleich $\mathrm{zu}$ den anderen Formen nur ein kleines Segment des Arbeitsmarktes (Ende 2010 knapp $3 \%$ der Gesamtbeschäftigung), hat aber seit ihrer Deregulierung durch die Hartz-Gesetze sehr kräftig zugenommen. Die hohen Zuwachsraten, die den Gesamtumfang in den 2000er Jahren ver- doppelten, sowie die erhöhten Prekaritätsrisiken im Vergleich zu Beschäftigten im Normalarbeitsverhältnis (NAV) und der eingetretene Funktionswandel (Seifert/ Brehmer 2008; Holst 2009, 2010) erklären das große Interesse der Öffentlichkeit an dieser Form. Sie ist in stärkerem Maße als andere Formen konjunkturabhängig. ${ }^{\text {? }}$

3 Diese Beschäftigungsformen gelten üblicherweise in Abgrenzung zum Normalarbeitsverhältnis (NAV) als atypisch (Keller/Seifert 2007). Nicht in die weiteren Betrachtungen einbezogen werden Freelancer, Ein-Euro-Jobber und Praktikanten sowie Solo-Selbstständige.

4 Die Datengrundlagen weisen Unterschiede hinsichtlich ihrer Fristigkeit auf. Während etwa für Leiharbeit lange Zeitreihen vorliegen, ist dies für Mini- und Midijobs nicht der Fall.

5 Die Daten enthalten eine unbekannte, Schätzungen zufolge auf mindestens ein Viertel zu veranschlagende Zahl von Studierenden und Rentnern, für die die Probleme der sozialen Sicherung weniger bedeutsam sein dürften.

6 Leih- und Zeitarbeit werden häufig synonym gebraucht. In Analogie zum Arbeitnehmerüberlassungsgesetz verwenden wir nur den Begriff Leiharbeit.

7 Der erheblichen Zunahme folgte 2008/2009 eine deutliche Abnahme, die bereits 2010 wieder in einen Zuwachs mündete und bis Oktober auf einen neuen Höchststand von 923.000 kletterte (BZA 2010). 


\subsection{GESAMTUMFANG UND STRUKTUR}

Der Gesamtumfang atypischer Beschäftigung lässt sich nicht einfach durch Addition der einzelnen Formen bestimmen, da Überlappungen auftreten können (z.B. Teilzeit und Befristung). Er ist seit den frühen 1990er Jahren von ca. $20 \%$ auf inzwischen mehr als ein Drittel der Gesamtbeschäftigung gestiegen (Brehmer/Seifert 2008). ${ }^{8}$ Von einem nur marginalen Segment, das in Analysen des Arbeitsmarktes vernachlässigt werden kann, kann also längst nicht mehr die Rede sein. Anders formuliert: Das NAV stellt den abnehmenden Regelfall, atypische Formen stellen den zunehmenden Ausnahmefall dar. Der Beschäftigungszuwachs im konjunkturellen Aufschwung zwischen 2005 und 2008 ging zu einem erheblichen Teil auf das Wachstum vor allem der Minijobs und der Leiharbeit zurück (Statistisches Bundesamt 2008). Ähnlich verlief die Entwicklung in der konjunkturellen Erholung 2010.

Daher beschreibt die Begrifflichkeit „Pluralisierung/Differenzierung der Beschäftigungsverhältnisse" die Entwicklung zutreffender als „Krise“ oder sogar „Erosion“ (für andere: Kommission für Zukunftsfragen 1996). Der langfristige Wandel der Beschäftigungsstrukturen und -formen wird sich wegen der zu erwartenden weiteren Tertiarisierung der Wirtschaftsstruktur sowie steigender Frauenerwerbsquoten fortsetzen, was aber nicht bedeutet, dass das NAV obsolet wird. Im Übrigen stellt in dieser Hinsicht die Bundesrepublik keinen Ausnahmefall dar: Unabhängig vom Typus des Wohlfahrtsstaates (sozialdemokratischer, konservativer oder liberaler Prägung) ist in den EU-Mitgliedstaaten eine Zunahme zu konstatieren (Schmid/Protsch 2009; Sengenberger 2010). ${ }^{9}$

Die sozialstrukturelle Zusammensetzung der Gruppe atypisch Beschäftigter (u.a. nach Alter, Geschlecht, Qualifikation, Branche) unterscheidet sich von der in NAV (zusammenfassend Bellmann et al. 2009). In sämtlichen Formen - mit Ausnahme der Leiharbeit - sind Frauen mehr (Teilzeit) oder weniger deutlich (Befristung) überrepräsentiert. Die Mehrzahl der Frauen $(57 \%)$ arbeitet in atypischen Beschäftigungsverhältnissen, sodass für sie von einem "neuen NAV“ gesprochen werden kann; die geschlechtsspezifische
Segmentation des Arbeitsmarktes (Sengenberger 1987) bzw. die Trennung in Insider und Outsider (Lindbeck/Snower 2001) und ihre langfristigen sozialen Konsequenzen werden durch die Ausweitung dieser Formen verstärkt.

In Bezug auf das Qualifikationsniveau sind Arbeitnehmer ohne abgeschlossene Berufsausbildung häufiger vertreten als solche mit Abschluss. Hinsichtlich des Alters sind alle Gruppen betroffen; allerdings sind jüngere Arbeitnehmer (15 bis 24 Jahre) mit befristeten oder Teilzeitverträgen überrepräsentiert. Weiterhin sind Ausländer aus Nicht-EU-Mitgliedsländern häufiger betroffen als Ausländer aus EUMitgliedsländern und Deutsche (Statistisches Bundesamt 2008).

\section{3 \\ Soziale Risiken atypischer Beschäftigung}

\subsection{PREKARITÄT - EINE DEFINITION}

Neben der bisher behandelten quantitativen Entwicklung spielen in der politischen und der wissenschaftlichen Diskussion die qualitativen Aspekte eine besondere Rolle. Die Begriffe atypische und prekäre Beschäftigung werden häufig synonym gebraucht (für andere: Dörre 2006). Atypisch bedeutet jedoch nicht notwendigerweise auch prekär (Rodgers 1989). ${ }^{10}$ Und umgekehrt schützen NAV nicht automatisch vor Prekarität. Die Diskussion über Prekarität versucht, die Entwicklungen des Arbeitsmarktes in einen breiteren soziologischen Kontext einzuordnen und eine Brücke zur Forschung über soziale Ungleichheit zu schlagen.

Zwei Ansätze lassen sich unterscheiden. Dörre (2006) und ähnlich Kraemer (2008) unterscheiden analytisch die beiden Handlungsebenen des Beschäftigungsverhältnisses und der Arbeits- und Berufstätigkeit. Die erste Ebene umfasst die Form des Arbeitskontraktes, das Einkommen sowie arbeits- und tarifrechtliche Rahmenbedingungen. Bei der zweiten Ebene geht es um „sinnhaft-subjektbezogene und sozialkommunikative Dimensionen der konkreten Arbeitstätigkeit" (Kraemer 2008, S. 80) wie Arbeitszufriedenheit, Gestaltungsmöglichkeiten der zeitlichen und sachlichen Arbeitsinhalte. Andere Akzente setzen Dörre et al. (2010), die in
Anlehnung an Bordieu und Castell den Prekaritätsbegriff vorrangig auf subjektive Bewertungen der Arbeitsbedingungen beziehen (ebenfalls Salengros et al.1989). Diese Dimension, die in diesem Ansatz den Kern des Prekaritätsbegriffs bildet, erweitern sie um die Kriterien Lohnhöhe und Beschäftigungsstabilität (Dörre et al. 2010), ohne sie näher zu bestimmen und zu operationalisieren. ${ }^{11}$ Vage bleibt auch die subjektive Prekaritätsdimension, die sich vor allem auf die empfundene Sicherheit des Arbeitsverhältnisses bezieht. Welche Variablen zugrunde gelegt und wie sie zu einem Indikator zusammengefügt werden, ist nicht erkennbar. Soziale Kontextfaktoren (wie Familieneinkommen, Beschäftigungsfähigkeit) bleiben unberücksichtigt.

Einen anderen, auf intersubjektiv nachvollziehbaren Kriterien beruhenden Ansatz schlägt Rodgers (1989) vor, der vier Dimensionen der Prekarität unterscheidet

- Beschäftigungsstabilität,

- Kontrolle über die Arbeitsbedingungen, - soziale Absicherung und Schutzrechte gegenüber Diskriminierung und unfairer Behandlung und

- Einkommen.

In Anlehnung an diesen Vorschlag formulieren und operationalisieren Keller/ Seifert (2007) folgende Kriterien zur Beschreibung und Bewertung von Prekarität:

8 Das Statistische Bundesamt beziffert für 2008 den Anteil atypischer Beschäftigung auf 22,2\%. Dieser geringere Wert kommt dadurch zustande, dass er nur Teilzeitbeschäftigte mit einer wöchentlichen Arbeitszeit von weniger als 21 Stunden einbezieht (Wingerter 2009)

9 Internationale Vergleiche sind schwierig - und daher selten, da die Abgrenzung der Formen unterschiedlich erfolgt.

10 Eine freiwillig gewählte, unbefristete Teilzeitbeschäftigung ist kurz- und mittelfristig nicht notwendigerweise prekär, wenn ein anderes Einkommen aus einem NAV die materielle Basis sichert; sie kann temporär die Vereinbarkeit von Familie und Beruf erleichtern. Ein langfristiges Problem besteht in der eigenständigen sozialen Sicherung im Alter.

11 Als prekär wird ein Erwerbsverhältnis definiert, "wenn es nicht dauerhaft oberhalb eines von der Gesellschaft definierten kulturellen Minimums existenzsichernd ist und deshalb bei den Möglichkeiten zur Entfaltung in der Arbeitstätigkeit, den sozialen Netzwerken, den politischen Partizipationschancen und der Fähigkeit zur Lebensplanung dauerhaft diskriminiert" (Dörre et al. 2010, S. 33). 


\begin{tabular}{|c|c|c|c|c|}
\hline \multicolumn{5}{|c|}{ Übersicht 1: Risiken der Prekarität } \\
\hline & Lohn & $\begin{array}{l}\text { Beschäftigungs- } \\
\text { stabilität }\end{array}$ & Weiterbildung & $\begin{array}{c}\text { Renten- } \\
\text { versicherung }\end{array}$ \\
\hline $\begin{array}{l}\text { befristete Voll- } \\
\text { zeitbeschäftigung }\end{array}$ & $\uparrow$ & $\uparrow$ & $\uparrow$ & $\uparrow$ \\
\hline unbefristete Teilzeit & $\uparrow$ & $\downarrow$ & $\uparrow$ & $\uparrow$ \\
\hline $\begin{array}{l}\text { befristete } \\
\text { Teilzeit }\end{array}$ & $\uparrow$ & $\uparrow$ & $\uparrow$ & $\uparrow$ \\
\hline $\begin{array}{l}\text { geringfügig, } \\
\text { unbefristet }\end{array}$ & $\uparrow$ & - & $\uparrow$ & $\uparrow$ \\
\hline $\begin{array}{l}\text { geringfügig, } \\
\text { befristet }\end{array}$ & $\uparrow$ & $\uparrow$ & $\uparrow$ & $\uparrow$ \\
\hline $\begin{array}{l}\text { Zeitarbeit/ } \\
\text { Leiharbeit }\end{array}$ & $\uparrow$ & $\uparrow$ & - & $\uparrow$ \\
\hline \multicolumn{5}{|c|}{$\begin{array}{l}\uparrow \text { höheres Risiko als bei NAV } \\
\downarrow \text { geringeres Risiko als bei bei NAV }\end{array}$} \\
\hline
\end{tabular}

- ein die materielle Existenz sicherndes Einkommen, welches, wie in der international-vergleichenden Forschung üblich, mindestens zwei Drittel des Medianeinkommens beträgt. Von Bedeutung ist weiterhin die Berücksichtigung des Haushaltskontextes bzw. die explizite Unterscheidung zwischen Individual- und Haushaltseinkommen, wobei vor allem gleichstellungspolitische Gründe für die zuerst genannte Variante sprechen;

- Ausmaß der Integration in die Systeme der sozialen Sicherung, vor allem die Rentenversicherung; ${ }^{12}$

- Beschäftigungsstabilität, die sich auf kontinuierliche Erwerbstätigkeit, d.h. auf ein möglichst ununterbrochenes Beschäftigungsverhältnis, nicht auf Arbeitsplatzsicherheit bezieht (employment security versus job security);

- Beschäftigungsfähigkeit (im Sinne von employability), operationalisiert vor allem durch den Zugang zu betrieblicher Weiterbildung und Gefährdungen der Gesundheit aufgrund belastender Arbeitsbedingungen.

Dieser Ansatz begreift Prekarität als mehrdimensionales Phänomen. Erste empirische Analysen tragen diesem Anspruch Rechnung und bewerten die Formen atypischer Beschäftigung anhand der genannten Kriterien (Brehmer/Seifert 2008). Die dynamische Perspektive kommt aber zu kurz, weil die entsprechenden Studien als Momentaufnahmen eine, zu einem bestimmten Zeitpunkt vorfindbare Beschäftigungssituation beleuchten. Insofern können auf beiden Konzepten beruhende Analysen nur bedingt Aussagen treffen über den Verbleib in prekärer Beschäftigung. Erforderlich wäre, die Chancen, einen als prekär eingestuften Status überwinden zu können (Aufwärtsmobilität), in die Betrachtungen ebenso einzubeziehen wie Angaben zu Prekaritätsphasen im Erwerbsverlauf.

\subsection{ERHÖHTE PREKARITÄTSRISIKEN}

Die mittlerweile zahlreichen empirischen Arbeiten zeigen, ${ }^{13}$ dass atypisch Beschäftigte deutlich höheren Prekaritätsrisiken die aber ebenfalls nicht frei von solchen Risiken sind, allerdings auf weitaus geringerem Niveau. Der Prekaritätsgrad ist nicht nur zwischen den Formen atypischer Beschäftigung abgestuft, sondern auch zwischen den Kriterien (Übersicht 1).

Beim Lohn sind atypische Beschäftigungsformen ohne Ausnahme benachteiligt (Giesecke/Groß 2007; Anger/Schmidt 2008; Kvasnicka 2008; Wingerter 2009; Schäfer 2010). Am stärksten ausgeprägt ist die Lohndiskriminierung bei geringfügiger Beschäftigung und bei Leiharbeit (Brehmer/Seifert 2008; Wingerter 2009).

Ähnliche Unterschiede bestehen bei der Beschäftigungsstabilität. Besonders instabil im Vergleich zu NAV ist Leiharbeit (Antoni/Jahn 2006; Kvasnicka/Werwatz 2006; Kvasnicka 2008; Bellmann/ Kühl 2008; Brenke 2008; Lehmer/Ziegler 2010), abgeschwächt fällt der Unterschied zwischen NAV und befristeter Beschäftigung aus (Boockmann/Hagen 2006; Brzinsky-Fay et al. 2007; Giesecke/Groß 2007). Für Teilzeitarbeit stellen Brehmer/ Seifert (2008) dagegen eine gegenüber allen anderen Formen höhere Beschäftigungsstabilität fest.

Atypisch Beschäftigte sind auch beim Zugang zu betrieblich-beruflicher Weiterbildung gegenüber vergleichbaren $\mathrm{Be}$ schäftigten mit NAV benachteiligt (Baltes/ Hense 2006; Brehmer/Seifert 2008). Besonausgesetzt sind als Arbeitnehmer in NAV, ders ausgeprägt ist die Diskriminierung bei geringfügiger Beschäftigung, abgeschwächt bei befristeter (Reinowski/Sauermann 2008) sowie bei Leiharbeit.

Einen Schritt über diese Partialanalysen hinaus geht eine Untersuchung, die auf dem Konzept des DGB-Index „Gute Arbeit" basiert und eine größere Zahl von Kriterien zu einem Indikator zusammenfasst (Seifert/Tangian 2009). Im Vergleich zu NAV schneidet befristete Beschäftigung deutlich schlechter ab und Leiharbeit erreicht nochmals geringere Werte. In diesen zusammengesetzten Indikator gehen vor allem arbeitsplatzbezogene Kriterien ein wie Grad der körperlichen Anforderungen oder Arbeitsintensität. Auch bei diesem Kriterium, das Hinweise auf gesundheitliches Gefährdungspotenzial (Lenhardt et al. 2010) und dadurch verursachte Risiken der Arbeits- und damit der Beschäftigungsfähigkeit liefert, werden Leiharbeit und befristete Beschäftigung schlechter bewertet als NAV. Die Chancen, die Arbeitsfähigkeit langfristig zu sichern, sind bei diesen Formen doppelt reduziert, da gleichzeitig die Qualifizierungsmöglichkeiten eingeschränkt sind. Für diesen wachsenden Personenkreis stehen die Chancen, bis zum Erreichen der gesetzlichen Ruhestandsgrenze im Erwerbsleben verbleiben zu können, sehr schlecht.

Diese skizzierten Prekaritätsrisiken können kumulieren. Das ist beispielsweise der Fall, wenn Beschäftigte ein geringeres Einkommen erhalten, instabil beschäftigt sind und nur über eingeschränkten Zugang zu betrieblicher Weiterbildung verfügen. Aufgrund geringer und zudem unsicherer Einkommen fällt es ihnen schwer, in Eigenregie durch berufliche Weiterbildung Qualifikationsdefizite auszugleichen und so Anschluss an sich ändernde Qualifikationsanforderungen zu halten, also ihre Beschäftigungsfähigkeit zu sichern. Unter

\footnotetext{
12 Wir diskutieren das Problem am Beispiel der Rentenversicherung. In der Krankenversicherung kann auf individueller Ebene Schutz atypisch Beschäftigter (aufgrund von Familienversicherung) gegeben sein; auf der kollektiven Ebene besteht das Problem der Erosion der Finanzierungsbasis, mit der Folge, dass weniger Beschäftigte mit NAV höhere Beiträge leisten müssen. Auch die häufig fehlenden Ansprüche an die Arbeitslosenversicherung stellen für atypisch Beschäftigte (vor allem bei Befristungen und Leiharbeit) ein Problem dar.

13 Vgl. besonders das Themenheft der Zeitschrift für Arbeitsmarktforschung "Atypische Beschäftigung und Niedrigeinkommen" und die dort angegebene Literatur (ZAF 2008).
} 
diesen Vorzeichen schwinden die Chancen auf Aufwärtsmobilität. In welchem Maße Prekaritätsrisiken sich verstärken und Aufwärtsmobilität beeinträchtigen, ist bislang empirisch nicht geklärt. Ebenso fehlt es an empirisch und theoretisch fundierten Erklärungen für die Mobilitätsbarrieren.

\subsection{LANGFRISTIGE KONSEQUENZEN}

Erstaunlicherweise finden die langfristigen Konsequenzen atypischer Beschäftigung bislang kaum Beachtung. Deren Nachteile reichen über die Phase der Erwerbstätigkeit hinaus und bestimmen die Einkommen in der Ruhestandsphase. Insofern betreffen die Probleme des Arbeitsmarktes auch die sozialen Sicherungssysteme, vor allem die Rentenversicherung. Infolge der niedrigeren Beiträge während der Erwerbstätigkeit (etwa während einer Teilzeittätigkeit oder als Minijobber) sowie eines größeren Beschäftigungsrisikos wächst die Wahrscheinlichkeit, dass die erworbenen Ansprüche nicht subsistenzsichernd sind (Steffen 2010). Ergänzende Sozialleistungen (Grundsicherung im Alter) werden notwendig, die aus dem allgemeinen Steueraufkommen finanziert werden müssen. Die Ausweitung atypischer Beschäftigungsverhältnisse erhöht individuell das Risiko der Altersarmut, kollektiv erodiert die Beitragsbasis der Rentenversicherung, zudem ändert sich die Struktur öffentlicher Ausgaben. Wie wir gezeigt haben, sind Frauen bei sämtlichen Formen (mit Ausnahme der Leiharbeit) überproportional betroffen. Deshalb tragen sie die größten Risiken zukünftiger Altersarmut.

Mit wachsenden Transferzahlungen steigt der Anteil konsumtiver Ausgaben an den öffentlichen Haushalten, bei gegebenen Budgets schrumpfen die Mittel für investive Verwendungen. Diese Umschichtungen dämpfen die Wachstumsdynamik. Eine ähnliche Wirkung lässt auch der eingeschränkte Zugang atypisch Beschäftigter zu betrieblicher Weiterbildung erwarten. Für einen wachsenden Teil der Beschäftigten wird es schwieriger, Wissen und Fertigkeiten an veränderte, aktuelle Qualifikationsanforderungen anzupassen. Sollten sich die beschriebenen Veränderungen im Erwerbssystem fortsetzen, drohen gravierende Konsequenzen für die Innovationsfähigkeit einer alternden Arbeitsgesellschaft.

Tabelle 2: Übergänge: aus Nichterwerbstätigkeit in Erwerbstätigkeit - in \% -

\begin{tabular}{l|cccccc}
\hline $\begin{array}{l}\text { Typ des alten } \\
\text { Arbeitsverhältnisses }\end{array}$ & Leiharbeit & befristet & $\begin{array}{c}\text { Typ des neuen Arbeitsverhältnisses } \\
\text { 400-Euro- } \\
\text { Job }\end{array}$ & $\begin{array}{c}\text { Teilzeit bis } \\
\text { 35 Stunden }\end{array}$ & $\begin{array}{c}\text { Normalarbeits- } \\
\text { verhältnis }\end{array}$ & $\begin{array}{c}\text { nicht } \\
\text { erwerbstätig }\end{array}$ \\
\hline Leiharbeit & 12 & 16 & 3 & 2 & 17 & 50 \\
befristet & 4 & 27 & 6 & 4 & 15 & 45 \\
400-Euro-Job & 2 & 12 & 25 & 9 & 5 & 47 \\
Teilzeit bis 35 Stunden & 3 & 16 & 11 & 19 & 10 & 41 \\
Normalarbeitsverhältnis & 3 & 13 & 3 & 3 & 41 & 37 \\
Insgesamt & 4 & 19 & $\mathbf{7}$ & $\mathbf{5}$ & $\mathbf{2 3}$ & $\mathbf{4 2}$ \\
\hline & & & & & WVI & MITTEILUNGEN
\end{tabular}

\subsection{EINGESCHRÄNKTE AUFWÄRTS- MOBILITÄT}

Die aufgezeigten Prekaritätsrisiken wären in ihren sozialen Wirkungen in dem Maße zu relativieren, wie atypische Beschäftigung als Einstieg in den Arbeitsmarkt und als nur kurzzeitige Durchgangsstation zu NAV dienen würde, wie es der Gesetzgeber bei der Verabschiedung der HartzGesetze erwartete. Wie Verlaufsanalysen zeigen, findet Aufwärtsmobilität jedoch nur begrenzt statt. Übergänge aus atypischer Beschäftigung in NAV gelingen bei Arbeitsplatzwechsel deutlich seltener als zwischen NAV. Von einer Brückenfunktion kann nicht die Rede sein.

Befristet Beschäftigte und Leiharbeitnehmer münden nach Verlust ihres Arbeitsplatzes, wenn man den Verbleib in Arbeitslosigkeit zunächst außer Acht lässt, überproportional häufig wieder in vergleichbar unsichere Beschäftigungsformen ein (Gensicke et al. 2010; Lehmer/ Ziegler 2010; Tabelle $2^{14}$ ). Vormals Teilzeitund geringfügig Beschäftigte weisen den geringsten Anteil an Personen auf, die bei Wiedereintritt in die Erwerbstätigkeit einem NAV nachgehen. Dieser Befund hat wesentlich damit zu tun, dass die überwiegende Mehrheit nicht an einem Wechsel in ein NAV interessiert sein dürfte. Mit einem Anteil von $17 \%$ kommt es bei ehemaligen Leiharbeitnehmern am häufigsten von allen vormals atypisch Beschäftigten zu einem Übergang in ein NAV, noch schlechter schneiden ehemals befristet Beschäftigte ab. Mit Abstand am häufigsten treten jedoch Personen, die aus einem NAV kommen, wieder in ein NAV ein (41\%).

Ähnlich unterscheiden sich die Risiken, nach Beendigung eines Arbeitsverhältnisses arbeitslos zu sein. Während von den Beschäftigten mit NAV 37 \% nicht den Wechsel in ein neues Arbeitsverhältnis schaffen, gelingt $50 \%$ der Leiharbeitnehmer und $45 \%$ der befristet Beschäftigten der Anschluss nicht. Dieser Befund ist deshalb erstaunlich, weil Leiharbeitnehmer erheblich seltener als Beschäftigte aus einem NAV Ansprüche auf Arbeitslosengeld I geltend machen können und deshalb einem stärkeren sozialen Druck zur Aufnahme einer neuen Beschäftigung ausgesetzt sind (Gensicke et al. 2010).

Diese empirischen Befunde liefern robuste Hinweise, dass eine beachtliche (relative) Pfadabhängigkeit in Bezug auf den Typ des Arbeitsverhältnisses besteht: So münden vormals atypisch Beschäftigte wiederum eher in atypische Beschäftigungsverhältnisse ein und Personen aus einem NAV eher wieder in ein NAV.

Auch bei der Einkommensmobilität zeigen sich starke Beharrungstendenzen. In einem Zeitraum von vier Jahren verbleiben fast zwei Drittel der Personen im unteren Einkommenssegment (Schäfer/ Schmidt 2009). Diese Quote ist in den letzten Jahren sogar gestiegen. Auf dem deregulierten Arbeitsmarkt fällt es schwerer, den Niedriglohnbereich zu verlassen und Anschluss an Aufwärtsmobilität zu finden. Offensichtlich bedeutet mehr Flexibilität nicht automatisch auch mehr Mobilität. Unklar bleibt, welche Faktoren mobilitätseinschränkend wirken.

14 Untersucht wurde, wie die Wechsel aus unterschiedlichen Beschäftigungsformen sowohl nach Kündigungen durch den Arbeitgeber als auch nach Eigenkündigungen in einem Zeitraum von zwei bis 14 Monaten verlaufen. 
Beschäftigungsverhältnisse über verschiedene Branchen verteilt. Vorschläge, die sich mehrheitlich um einen moderaten Stundenlohn zwischen 7,50 und 8,50€ bewegen und daher faktisch kaum die häufig befürchteten Beschäftigungsverluste zur Folge haben dürften, liegen seit Jahren vor. Im Übrigen ist Deutschland eines der wenigen EU-Mitgliedsländer ohne entsprechende Regelungen. Diese Tatsache kann die bereits bestehenden Probleme (vor allem bei Leiharbeit) verschärfen, wenn ab Mai 2011 die Freizügigkeitsregelungen der EU für Arbeitnehmer aus den MOEStaaten gelten, die 2004 der EU beigetreten sind. Eine entsprechende Änderung des Arbeitnehmerüberlassungsgesetzes erweist sich als notwendig. ${ }^{18}$

(2) Das Prinzip „gleicher Lohn für gleiche Arbeit" bei Normal- und atypischen Beschäftigungsverhältnissen sollte nicht nur als Anspruch formuliert, sondern müsste in der Praxis tatsächlich durchgesetzt werden. Der Grundsatz des „equal pay“19 für Stamm- und andere Arbeitnehmer des Entleihbetriebs sollte nicht erst nach einer gewissen Einarbeitungszeit, sondern von Beginn der Tätigkeit an gelten, da er sonst bei bestimmten Formen (besonders bei Leiharbeit aufgrund der häufig kurzen Einsatzzeiten) seine intendierte Wirkung weitgehend verfehlt.

(3) Atypische Beschäftigungsverhältnisse sind (mit der Ausnahme regulärer Teilzeit) weniger stabil als NAV. Die Einführung einer sog. Risikoprämie würde das (vor allem bei befristeter Beschäftigung) im Vergleich zum NAV erhöhte Risiko des Arbeitsplatzverlustes und des damit verbundenen Einkommensverlustes zwar nicht eliminieren können, zumindest aber finanziell kompensieren. ${ }^{20}$ Diese Prämie könnte durchgängig als Zuschlag zum regulären Entgelt oder als Pauschale bei Eintritt des Risikos gezahlt werden. Derartige Regelungen bestehen bereits in den Rechtsordnungen einiger EU-Mitgliedsländer (Frankreich, Portugal).

(4) Beschäftigungsstabilität ist perspektivisch weiter gefasst als Arbeitsplatzsicherheit (im Sinne von job security auf dem betriebsinternen Markt) und als ununterbrochene Beschäftigung zu verstehen, einschließlich der Chancen auf Wieder- bzw. Neubeschäftigung bei Verlust des Arbeitsplatzes. Voraussetzung ist die Sicherung der Beschäftigungsfähigkeit (im Sinne von employability auch auf dem externen Markt). Sie hängt vor allem in Zeiten raschen qualifikatorischen Wandels und abnehmender Halbwertzeiten des erworbenen Wissens nicht nur von der beruflichen Erstausbildung, sondern wesentlich von der beruflichen Weiterbildung ab. Aufgrund der bei atypisch Beschäftigten eingeschränkten Zugangsmöglichkeiten zu betrieblicher Weiterbildung drohen mittel- und langfristig sich verschärfende Mismatch-Probleme sowie eine Innovationen und wirtschaftliches Wachstum behindernde Unterinvestition in Humankapital. In Anbetracht des befürchteten Fachkräftemangels ist die Einführung generalisierter, individueller Ansprüche oder deren Finanzierung aus einem Fonds auf gesetzlicher und/oder tarifvertraglicher Basis zu empfehlen.

(5) Angesichts der durch die Ausweitung atypischer Beschäftigung verursachten sozialen Probleme, die über die Erwerbsphase hinausreichen, dürfen Arbeitsmarkt- und Sozialpolitik nicht mehr wie bisher üblich getrennt behandelt werden. Vielmehr sind die Systeme der sozialen Sicherung (Arbeitslosigkeit, Krankheit und vor allem Alter) aufeinander abzustimmen, um das mehrere Jahrzehnte als gelöst angesehene, künftig jedoch wieder auftretende Probleme der Altersarmut nicht zu verschärfen. Es betrifft vor allem Mini- und Midijobber, aber auch regulär Teilzeitbeschäftigte. Re-Regulierung zielt darauf, das Risiko zu mindern, im Alter zusätzliche, aus dem

15 Die Bundesregierung will laut Koalitionsvertrag eine „Erhöhung und Dynamisierung der Grenze sozialversicherungsfreier Beschäftigungsverhältnisse" durchsetzen.

16 Eine solche Balance versuchen die Konzepte von Flexicurity herzustellen (Keller/Seifert 2008).

17 So sollte das Abgabenprivileg der Minijobs bei den Beiträgen zur Sozialversicherung, wie vom Sachverständigenrat seit Jahren gefordert (SVR 2008, S. 421ff.) abgeschafft werden, sämtliche Beschäftigungsformen sollten hinsichtlich ihrer Sozialversicherungspflicht gleich behandelt werden

18 Das Bundesarbeitsministerium plant einen entsprechenden Vorstoß. Vgl. zu diesem Thema auch Siebenhüter in diesem Heft.

19 In der Stahlindustrie besteht seit Herbst 2010 ein Flächentarifvertrag mit equal pay-Regelungen für Leiharbeitnehmer.

20 Lohntheoretische Überlegungen rechtfertigen (bei perfektem Wettbewerb) Lohndifferentiale für Tätigkeiten mit unterschiedlichen Risiken (Cahuc/ Zylberberg 2001). 
Steueraufkommen zu finanzierende Transferleistungen (Hartz IV) in Anspruch nehmen zu müssen. Empfohlen wird der Umbau der Rentenversicherung zu einem dreistufigen System, wie es in der Schweiz existiert, das aus einer nicht beitrags-, sondern steuerfinanzierten Mindestsicherung, einem wie bisher beitragsfinanzierten Teil sowie einer freiwilligen Zusatzversicherung besteht. Das Implementationsproblem besteht darin, dass atypisch Beschäftigte aufgrund ihrer niedrigen Einkommen einerseits kaum Beiträge zahlen bzw. Ansprüche erwerben und andererseits keine freiwilligen Zusatzleistungen erbringen können. Daher wäre ein grundlegender
Systemwechsel im Sinne der Einführung einer steuerfinanzierten, vom vorherigen Erwerbsstatus unabhängigen, lediglich den Bürgerstatus voraussetzenden Basis- bzw. Grundsicherung im Alter eine mögliche Konsequenz; zu deren Finanzierung wären nicht nur Arbeits-, sondern sämtliche Einkommensformen heranzuziehen.

\section{LITERATUR}

Anger, C./Schmidt, J. (2008): Gender Wage Gap und Familienpolitik, in: IW Trends 35 (2), S. 55-68

Antoni, M./Jahn, E. (2006): Boomende Branche mit hoher Fluktuation. Mit der Flexibilisierung des Arbeitnehmerüberlassungsrechts in den letzten Jahren wurden die Beschäftigungsverhältnisse der Leiharbeiter immer kürzer, IAB-Kurzbericht (14), Nürnberg

Baltes, K./Hense, A. (2006): Weiterbildung als Fahrschein aus der Zone der Prekarität?, hrsg. vom Rat für Sozial- und WirtschaftsDaten (RatSWD), Working Paper (4)

Bellmann, L./Kühl, A. (2008): Expansion der Leiharbeit?, Edition der Hans-Böckler-Stiftung (224), Düsseldorf

Bellmann, L./Fischer, G./Hohendanner, Chr. (2009): Betriebliche Dynamik und Flexibilität auf dem deutschen Arbeitsmarkt, in: Möller, J./ Walwei, U. (Hrsg.): Handbuch Arbeitsmarkt 2009, Nürnberg,

S. 360-401

Boockmann, B./Hagen, T. (2006): Befristete Beschäftigungsverhältnisse - Brücken in den Arbeitsmarkt oder Instrumente der Segmentierung?, Baden-Baden

Brehmer, W./Seifert, H. (2008): Sind atypische Beschäftigungsverhältnisse prekär? Eine empirische Analyse sozialer Risiken, in: Zeitschrift für Arbeitsmarktforschung 41 (4), S. 501-531

Brenke, K. (2008): Leiharbeit breitet sich rasant aus, in: DIW-Wochenbericht (19), S. 242-252

Brzinsky-Fay, C./Protsch, P./Schulze Buschoff, K. (2007): Atypische Beschäftigung - Umfang, Dynamik und soziale Sicherung im europäischen Vergleich, Arbeitspapier, vorgelegt zur 5. Internationalen Forschungskonferenz über soziale Sicherheit, Warschau

Bundesministerium für Arbeit und Soziales (BMAS) (2006):

Die Wirksamkeit moderner Dienstleistungen am Arbeitsmarkt. Bericht 2006 des Bundesministeriums für Arbeit und Soziales zur Wirkung der Umsetzung der Vorschläge der Kommission Moderne Dienstleistungen am Arbeitsmarkt (ohne Grundsicherung für Arbeitsuchende), Berlin

Bundesverband Zeitarbeit (BZA) (2010): http://www.bza.de/fileadmin/ bilder/2010/1010_Zeitarbeitsindex.pdf

Cahuc, P./Zylberberg, A. (2001): Labor Economics, Cambridge/London Destatis (Statistisches Bundesamt Deutschland) (Hrsg.) (2009): http:// www.destatis.de/jetspeed/portal/cms/Sites/destatis/Internet/DE/Presse/ pm/2009/08/PD09_304_132,templateld=renderPrint.psml

Dörre, K. (2006): Prekäre Arbeit. Unsichere Beschäftigungsverhältnisse und ihre sozialen Folgen, in: Arbeit 15 (1), S. 181-193

Dörre, K./Franz, K./Holst, H. (2010): Einschätzungen zum Forschungsstand Prekarität, in: Beiträge zur Arbeitspolitik und Arbeitsforschung, hrsg. von der IG Metall, Frankfurt/M., S. 32-44
Gensicke, M./Herzog-Stein, A./Seifert, H./Tschersich, N. (2010): Einmal atypisch, immer atypisch beschäftigt? Mobilitätsprozesse atypischer und normaler Arbeitsverhältnisse im Vergleich, in: WSI-Mitteilungen 63 (4), S. 179-187, download unter: http://www.boeckler.de/119_103164.html Giesecke, J./Groß, M. (2007): Flexibilisierung durch Befristung. Empirische Analysen zu den Folgen befristeter Beschäftigung, in: Keller, B./ Seifert, H. (Hrsg.): Atypische Beschäftigung - Flexibilisierung und soziale Risiken, Berlin, S. 85-105

Holst, H. (2009): Disziplinierung durch Leiharbeit? Neue Nutzungsstrategien von Leiharbeit und ihre arbeitspolitischen Folgen, in: WSIMitteilungen 62 (3), S. 143-149, download unter: http://www.boeckler. de/119_94379.html

Holst, H. (2010): „Die Flexibilität unbezahlter Zeit" - Die strategische Nutzung von Leiharbeit, in: Arbeit 19 (2+3), S. 164-177

Keller, B./Seifert, H. (Hrsg.) (1995): Atypische Beschäftigung. Verbieten oder gestalten?, Köln

Keller, B./Seifert, H. (Hrsg.) (2007): Atypische Beschäftigung. Flexibilisierung und soziale Risiken, Berlin

Keller, B./Seifert, H. (2008): Flexicurity: Ein europäisches Konzept und seine nationale Umsetzung, Expertise für die Friedrich-Ebert-Stiftung, Bonn Kommission für Zukunftsfragen der Freistaaten Bayern und Sachsen (1996): Erwerbstätigkeit und Arbeitslosigkeit in Deutschland, Bonn

Kraemer, K. (2008): Prekarität - was ist das?, in: Arbeit 17 (2), S. 77-90 Kvasnicka, M. (2008): Does Temporary Help Work Provide a Stepping Stone to Regular Employment?, NBER Discussion Paper w13843, hrsg. vom National Bureau of Economic Research, Cambridge

Kvasnicka, M./Werwatz, A. (2006): Lohneffekte der Zeitarbeit,

in: Bundesarbeitsblatt 2, S. 2-10

Lenhardt, U./Ertel, M./Morschhäuser, M. (2010): Psychische Arbeitsbelastungen in Deutschland: Schwerpunkte - Trends - betriebliche Umgangsweisen, in: WSI-Mitteilungen 63 (7), S. 335-342, download unter: http://www.boeckler.de/119_107747.html

Lehmer, F./Ziegler, K. (2010): Brückenfunktion der Leiharbeit. Zumindest ein schmaler Steg, IAB-Kurzbericht (13), Nürnberg

Lindbeck, A./Snower, D. J. (2001): Insiders versus outsiders, in: Journal of Economic Perspective 15 (1), S. 165-188

Neubäumer, R./Tretter, D. (2008): Mehr atypische Beschäftigung aus theoretischer Sicht, in: Industrielle Beziehungen 15 (3), S. 256-278 Nienhüser, W. (2007): Betriebliche Beschäftigungsstrategien und atypische Arbeitsverhältnisse, in: Keller, B./Seifert, H. (Hrsg.): Atypische Beschäftigung. Flexibilisierung und soziale Risiken, Berlin, S. 45-65 Reinowski, E./Sauermann, J. (2008): Befristete Beschäftigung, berufliche Weiterbildung und ihre Finanzierung, hrsg. vom Rat für Sozial- und WirtschaftsDaten (RatSWD), Working Paper (14) 
Rodgers, G. (1989): Precarious work in Western Europe: The state of the debate, in: Rodgers, G./Rodgers, J. (Hrsg.): Precarious jobs in labour market regulation: The growth of atypical employment in Western Europe, Brussels, S. 1-16

Salengros, P./van de Leemput, C./Mukibangiey, L. (1989): Psychological and sociological perspectives on precarious employment in Belgium, in: Rodgers, G./Rodgers, J. (Hrsg.): Precarious jobs in labour market regulation: The growth of atypical employment in Western Europe, Brussels, S. $197-223$

Sachverständigenrat zur Begutachtung der gesamtwirtschaftlichen Entwicklung (SVR) (2008): Jahresgutachten 2008/2009. Die Finanzkrise meistern - Wachstumskräfte stärken, Wiesbaden

Schäfer, H. (2010): Sprungbrett oder Sackgasse? - Entwicklung und Strukturen von flexiblen Erwerbsformen in Deutschland, in: IW-Trends 37 (1), S. 47-63

Schäfer, H./Schmidt, J. (2009): Einkommensmobilität in Deutschland Entwicklung, Strukturen, Determinanten, in: IW-Trends 36 (2), S. 91-105 Schmid, G./Protsch, P. (2009): Wandel der Erwerbsformen in Deutschland und Europa, hrsg. vom Wissenschaftszentrum Berlin für Sozialforschung, Discussion Paper SP I (505), Berlin

Seifert, H./Brehmer, W. (2008): Leiharbeit : Funktionswandel einer flexiblen Beschäftigungsform, in: WSI-Mitteilungen 61 (6), S. 335-341, download unter: http://www.boeckler.de/169_94120.html

Seifert, H./Tangian, A. (2009): Index "Qualität der Arbeit" : Nordische Länder und Deutschland im Vergleich, in: WSI-Mitteilungen 62 (1),

S. 52-57, download unter: http://www.boeckler.de/169_94120.html
Sengenberger, W. (1987): Struktur und Funktionsweise von Arbeitsmärkten, Frankfurt (Main)/New York

Sengenberger, W. (2010): Beyond the measurement of unemployment and underemployment: The case for extending and amending labour market statistics, Ms. Geneva

Sesselmeier, W. (2007): (De)Stabilisierung der Arbeitsmarktsegmentation? Überlegungen zur Theorie atypischer Beschäftigung, in: Keller, B./ Seifert, H. (Hrsg.): Atypische Beschäftigung. Flexibilisierung und soziale Risiken, Berlin, S. 67-80

Statistisches Bundesamt (2008): Atypische Beschäftigung auf dem deutschen Arbeitsmarkt, Wiesbaden

Steffen, J. (2010): Gebrochene Erwerbsbiografien, atypische Beschäftigung und drohende Altersarmut, hrsg. von der Arbeitnehmerkammer, in: Perspektiven solidarischer Sicherungskonzepte (12), S. 1-11

Waltermann, R. (2010): Abschied vom Normalarbeitsverhältnis? Welche arbeits- und sozialrechtlichen Regelungen empfehlen sich im Hinblick auf die Zunahme neuer Beschäftigungsformen und die wachsende Diskontinuität von Erwerbsbiographien? Gutachten für den 68. Deutschen Juristentag, Bonn

Wingerter, C. (2009): Der Wandel der Erwerbsformen und seine Bedeutung für die Einkommenssituation Erwerbstätiger, in: Wirtschaft und Statistik (11), Wiesbaden, S. 1080-1098

Zeitschrift für Arbeitsmarktforschung (ZAF) (2008): Themenheft Atypische Beschäftigung und Niedrigeinkommen 41 (4) 